\title{
Influence Factors of Understanding Business Process Models
}

\author{
Jan Mendling ${ }^{1}$ and Mark Strembeck ${ }^{2}$ \\ 1 BPM Cluster, Faculty of Information Technology \\ Queensland University of Technology, Australia \\ j.mendling@qut.edu. au \\ 2 New Media Lab, Institute of Information Systems \\ Vienna University of Economics and Business Administration, Austria \\ mark.strembeck@wu-wien.ac . at
}

\begin{abstract}
The increasing utilization of business process models both in business analysis and information systems development raises several issues regarding quality measures. In this context, this paper discusses understandability as a particular quality aspect and its connection with personal, model, and content related factors. We use an online survey to explore the ability of the model reader to draw correct conclusions from a set of process models. For the first group of the participants we used models with abstract activity labels (e.g. A, B, C) while the second group received the same models with illustrative labels such as "check credit limit". The results suggest that all three categories indeed have an impact on the understandability.
\end{abstract}

\section{Introduction}

Even though workflow and process modeling have been used extensively over the past 30 years, we know surprisingly little about the act of modeling and which factors contribute to a "good" process model in terms of human understandability. This observation contrasts with the large body of knowledge that is available for the formal analysis and verification of desirable properties, in particular for Petri nets. To guarantee a certain degree of design quality of the model artifact in a wider sense, several authors propose guidelines for the act of modeling $[1,2]$ but yet with little impact on modeling practice. Clearly, an empirical research agenda is required for acquiring new insights into quality [3] and usage aspects [4] of process modeling.

Following this line of argument, a recent empirical study provides evidence that larger, real-world process models tend to have more formal flaws (such as deadlocks) than smaller models $[5,6]$. One obvious hypothesis related to this phenomenon would be that human modelers lose track of the interrelations of large and complex models due to their limited cognitive capabilities [7], and then introduce errors that they would not insert in a small model. There are other factors beyond size that presumably affect the understandability of a process model such as the degrees of sequentiality, concurrency, or structuredness [8]. 
Validating such hypothetical relationships empirically would not only represent a major step forward towards understanding quality of process models beyond verification, but also provide a sound theoretical basis for defining guidelines for process modeling in general.

Since only little research has been conducted on quality aspects of process models so far [3,9], we approach this area with an experimental design focusing on the understandability of process models (not of process modeling languages). By having a online questionnaire filled out, we aim to gain insight into empirical connections between personal and model characteristics and the ability of a person to understand a process model properly. In particular, we want to find out how the textual content of the activity labels might influence understandability. Figures 1 and 2 show two process model variants that were included in the questionnaire.

Our contribution related to process model understandability is twofold. First, we operationalize understandability and identify three categories of factors, i.e. personal, structural, and textual, that potentially influence model understandability. Second, we present the findings from an experiment that tested the relative importance of these factors. Indeed, all three categories appear to be relevant according to a logistic regression model for the experiment data. Against this background, the remainder of the paper is structured as follows. In Section 2 we discuss related work and identify a lack of empirically validated insight on the understandability of process models. Then, Section 3 introduces the research design, in particular, the conceptualization of the questionnaire and the data that we gather. In Section 4 we present the results of the statistical analysis. Section 5 concludes the paper, discusses limitations of the findings, and identifies open questions that need to be addressed by future research.

\section{Related Work on Understandability}

There are basically three streams of research related to our work in the conceptual modeling area: top-down quality frameworks, bottom-up metrics related to quality aspects, and empirical surveys related to modeling techniques.

One prominent top-down quality framework is the SEQUAL framework [10, 11]. It builds on semiotic theory and defines several quality aspects based on relationships between a model, a body of knowledge, a domain, a modeling language, and the activities of learning, taking action, and modeling. In essence, syntactic quality relates to model and modeling language; semantic quality to model, domain, and knowledge; and pragmatic quality relates to model and modeling and its ability to enable learning and action. Although the framework does not provide an operational definition of how to determine the various degrees of quality, it has been found useful for business process modeling in experiments [12].

The Guidelines of Modeling (GoM) [2] define an alternative quality framework that is inspired by general accounting principles. The guidelines include the six principles of correctness, clarity, relevance, comparability, economic ef- 


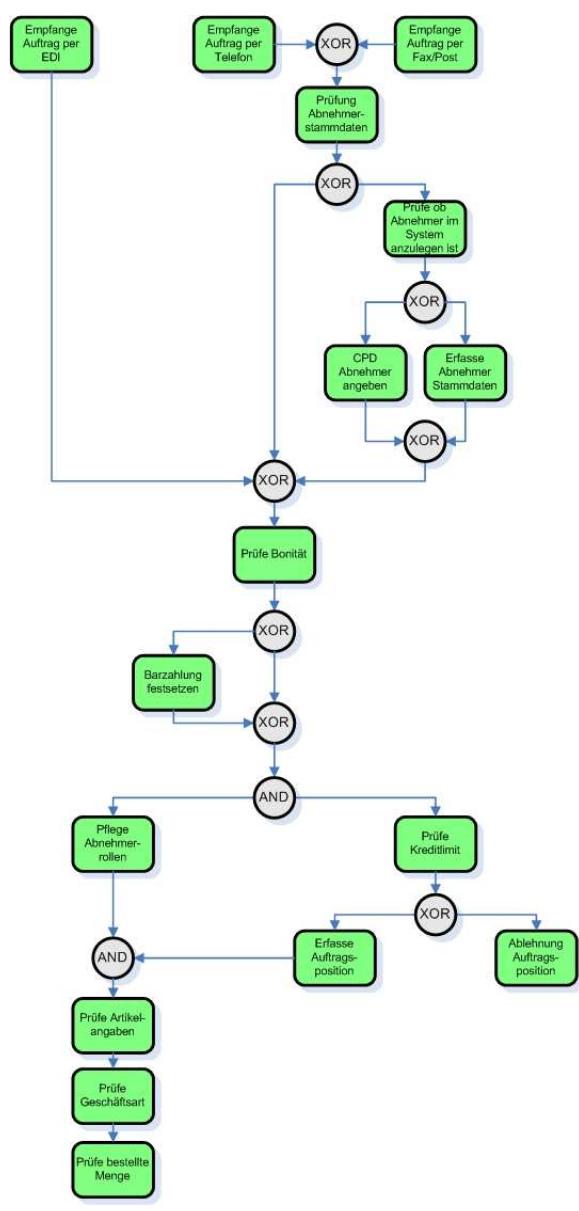

Fig. 1. Model 4 with Text (in German)

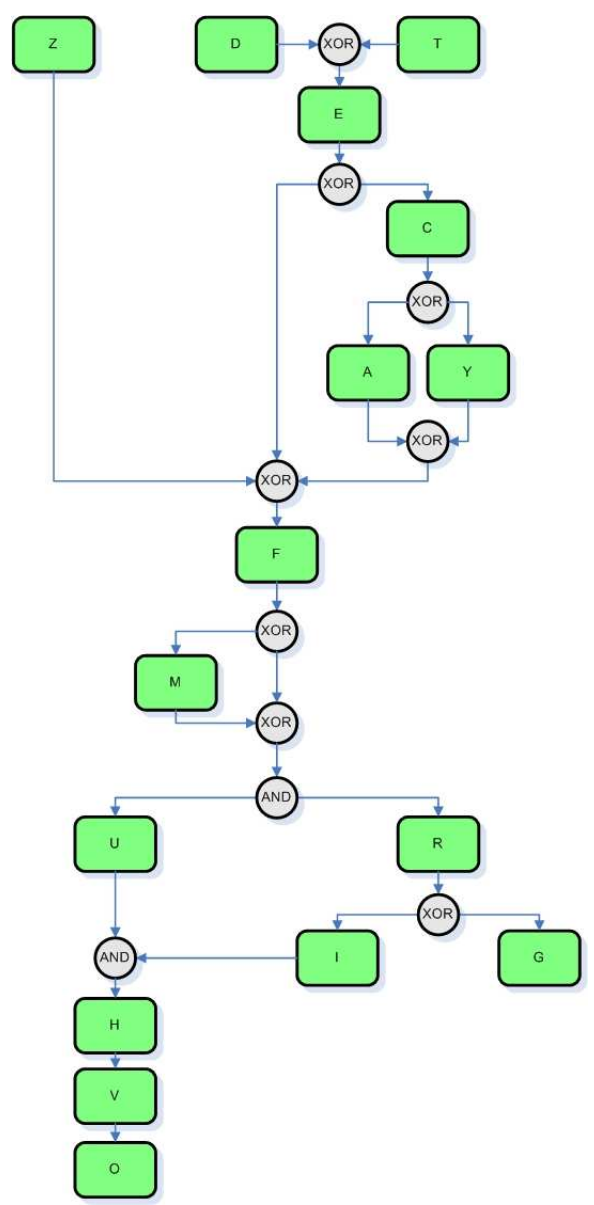

Fig. 2. Model 4 with Letters

ficiency, and systematic design. This framework was operationalized for Eventdriven Process Chains (EPCs) and also tested in experiments [2]. Furthermore, there are authors [3] advocating a specification of a quality framework for conceptual modeling in compliance with the ISO 9126 standard for software quality [13]. A respective adaptation to business process modeling is reported in [14]. Our experiment addresses partial aspects of these frameworks. In particular, we focus on understandability of process models as an enabler of pragmatic quality (SEQUAL) and clarity (GoM). This requires us not only to ask about understandability, but also check whether models are interpreted correctly. This is in line with research of [15] who experimented on conclusions that people can draw from models. In a more general design setting, the work on cognitive dimensions stresses understanding as one important interaction of a user with a model [16]. 
Several authors have published work on bottom-up metrics related to quality aspects of process models, stemming from different research and partially isolated from each other [17-25], or see [8] for an overview. Several of these contributions are theoretic without empirical validation. Most authors doing experiments focus on the relationship between metrics and quality aspects: [23] study the connection mainly between count metrics - for example, the number of tasks or splits - and maintainability of software process models; [26] validates the correlation between control flow complexity and perceived complexity; and $[6,8]$ use metrics to predict control flow errors such as deadlocks in process models. The results reveal that an increase in size of a model appears to have a negative impact on quality. This finding has an impact on the design of our questionnaire. To gain insights that are independent of process size, we keep the number of tasks constant and study which other factors might have an impact on understandability.

Finally, there are some empirical surveys related to modeling techniques. [27] study how business process modeling languages have matured over time. While this is valuable research it does not reveal insights on single, concrete process models. The same holds for [28] who study the usability of UML. [29] approach understandability, not of individual process models, but on the level of the modeling language. They find that EPCs seem to be more understandable than Petri nets. Inspired by this survey we decided to use an EPC-like notation in our questionnaire to minimize the impact of the notation on understandability.

To summarize, there is essentially one relation that seems to be confirmed by related research, and that is that larger models tend to be negatively connected with quality. The aim of our questionnaire is to enhance this rather limited body of knowledge.

\section{Research Design}

Related to understandability, we identify the following six research questions related to the factors that might influence understandability of process models $[29,8,30,11,9]$ :

1. What personal factors (beyond general psychological and intellectual factors) have an influence?

2. Which model characteristics (e.g. number and type of splits) contribute to a good understandability?

3. How is understandability related to the textual content that is described in the model?

4. How does the modeling purpose (e.g. documentation versus enactment) relate to understandability?

5. Which differences in understandability exist when observing semantically equivalent models described in different modeling languages?

6. What is the impact of different visual layout strategies or graph drawing algorithms on understandability? 
We approach these questions with an experimental design focusing on personal, model, and content characteristics (question 1, 2, and 3). Furthermore, we strive to neutralize the influence of the other factors: related to question 4, we gathered a set of process models from practice that capture different domains such as order processing and price calculation. All models were created for documentation purposes (question 4). Based on the observation by [29] that EPCs appear to be easier to understand than Petri nets, we chose an EPC-like notation without events (question 5). The participants received a short informal description of the semantics similar to $[31$, p.25]. Finally, we drew all models in the same top-to-bottom style with the start element at the top and end element at the bottom (question 6).

The experiment was conducted in three phases. First, we collected a set of six process models from practice that could be displayed on an A4 page. For each of these models we constructed a variant where the activity labels were replaced by capital letters as identifiers. The models were similar to model 4 depicted in Figures 1 and 2. For the 6 models we identified 6 yes/no questions related to the structure and the behavior specified by the model. These questions together with questions on personal experience and knowledge of process modeling were packed into two variants of the questionnaire, one for models with textual activity labels, one for models with letters. Second, we developed a website for conducting the survey as an online questionnaire. We chose an online questionnaire to get practitioners with modeling experience involved more easily. Furthermore, we were able to record the answer times, randomly define the presentation order of the 6 models, and we could randomly assign a questionnaire variant to the participant. Participation was voluntary. As an incentive the participants received feedback about their performance. Finally, the data was analyzed using the statistical software package SPSS.

\subsection{Data gathered in the Survey}

In the survey we gathered the following data related to the participants:

- THEORY: The participants had to answer six theoretical yes/no questions without before seeing the models about selected topics related to process modeling such as choices, concurrency, loops, and deadlocks. THEORY captures the sum of correct answers to these questions.

- DURATION: The participants were asked for how long they have been involved with business process modeling. The variable was measured ordinally on four levels: less than one month, less than a year, less than three years, and longer than three years.

- INTENSITY: The participants had to indicate how often they work with process models. There were four options to answer: daily, monthly, less frequent than monthly, never.

- TIME: This variable measures the time that the participants invested in answering the questionnaire.

- TEXT: This variable indicates whether the activities had textual labels (value 1) or only abstract letters (value 0 ). 
- PSCORE: This variable is calculated based on the answers given by the participant to the model related questions. It captures the number of correct answers by the person. The maximum value is 36 for six questions on six models. This variable serves as an operationalization of understandability related to a person.

Furthermore, we calculated some model metrics from the set proposed by [8]. These include:

- SIZE: This variable refers to the number of nodes of the process model graph.

- DIAMETER gives the length of the longest path from a start node to an end node in the process model.

- STRUCTUREDNESS of the process graph is one minus the number of nodes in structured blocks divided by the number of nodes.

- SEPARABILITY relates the number of cut-vertices to the number of nodes.

- TOKEN SPLIT sums up all concurrent threads that can be activated by ANDsplits and OR-splits in the process.

- CYCLICITY relates number of nodes on cycles to all nodes.

- HETEROGENEITY gives the type entropy of the connectors.

- SOUND indicates whether the process model is sound according to [32].

- MSCORE: This variable is calculated based on the answers given by the participants to the model related questions. It captures the sum of correct answers for this model. This variable serves as an operationalization of understandability related to a model.

Finally, we also measured aspects related to the textual labels of the model and correct answers to individual questions:

- TEXTLEngth gives the string length of all textual activity labels in the process model.

- CORRECTANSWER captures for each individual question answered by a participant whether it was answered correctly (value 1) or not (value 0). This variable serves as an operationalization of understandability related to a model aspect.

We distributed the link to the experiment via the German mailing lists EMISA and WI as well as among students that followed courses on process modeling at the Vienna University of Economics and Business Administration. Typically both academics and practitioners with an interest in conceptual modeling and information systems development are registered with these lists. The questionnaire was started by 200 persons and completed by 46 . From these 46 we excluded 4 people who spent less than 10 minutes (TIME) on the questionnaire since we assumed that to be the minimum time to provide meaningful answers. These 42 persons and their answers to the 36 questions establish the sample for our statistical analysis below. Altogether, 1512 answers are recorded in the sample. $65 \%$ of the participants had more than three years experience in process modeling. 


\subsection{Hypothetical Relations between Factors and Understandability}

Before conducting the statistical analysis we make hypothetical connections between the different variables explicit. In particular, we identify hypotheses related to personal factors, model factors, and content factors:

P1 A higher PSCORE of participants should be connected with higher values in THEORY, DURATION, INTENSITY, and TIME.

M1 A higher MSCORE of models should be associated with lower values in SIZE, DIAMETER, TOKEN SPLIT, and HETEROGENEITY since these metrics might indicate that the model is easier to comprehend.

M2 A higher MSCORE of models should be connected with higher values in STRUCTUREDNESS, SEPARABILITY, and SOUND since these metrics might be associated with models that are easier to comprehend.

C1 A higher sum of CORRECTANSWER should be connected with abstract labels (value of 0 in TEXT), basically our questions refer to structural properties of the model.

C2 A Correctanswer (value of 1) should be connected with a lower value in TEXTLENGTH, since it becomes harder to match the elements mentioned in the question with the elements in the graphical model.

In the following section we will use statistical methods to assess these hypotheses.

\section{Results}

In this section we present the results of our survey. First, we discuss the distribution of personal, model, and content factors as well as their correlation with the corresponding score. Then, we use logistic regression to gain insight into the relative importance of the different factors for predicting whether a question would be answered correctly.

\subsection{Personal Factors}

Figure 3 gives an overview of the PSCORE that the different participants achieved in the survey. The mean value was 25.21 for 36 questions which means that on average $70 \%$ of the questions were answered correctly. The best participant had 34 questions correct and two questions wrong. The correlation with some variables of P1, i.e. DURATIOn, InTENSITY, and TIME, was weakly positive, but not significant. In contrast to that, the Pearson correlation between PSCORE and THEORY was positive and significant $(\mathrm{p}=0.01)$ with a value of 0.491 . This might indicate that theoretical process modeling knowledge helps to answer questions correctly. Furthermore, THEORY was positively and significantly $(\mathrm{p}=0.01)$ correlated with INTENSITY of process modeling experience (0.438). 


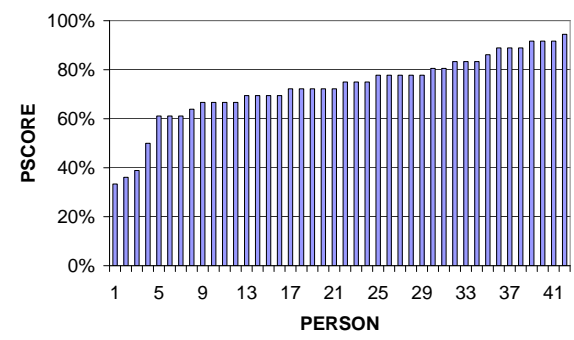

Fig. 3. pscore of participants

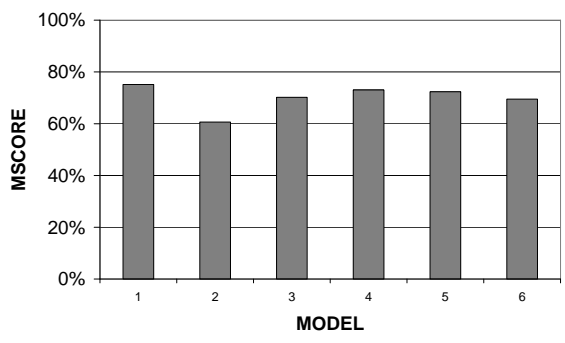

Fig. 4. mscore of model

\subsection{Model Factors}

Figure 4 gives an overview of the MSCORE that the different participants achieved per model. The mean percentage was $70 \%$ across the models. The model with the lowest MSCORE had on average $60 \%$ correct answers. This model had loops and parallel execution paths. From the variables mentioned in M1 and M2 only SEPARABILITY had a significant correlation according to Spearman with MSCORE of $0.886(\mathrm{p}=0.019)$. This strongly confirms the hypothetical impact direction of M2. The other variables showed a direction of correlation as expected, but without a sufficient significance. As an exception, structuredness had zero correlation in our sample.

\subsection{Content Factors}

Table 1 gives an overview of the sum of CORRECTANSWER disaggregated by TEXT. This table directly relates to hypothesis $\mathbf{C 1}$. The difference between both questionnaire types is quite narrow, such that the hypothesis cannot be confirmed. Furthermore, we calculated the correlation between CORRECTANSWER and textlength. The Pearson correlation coefficient of -0.836 on a significance level above 0.01 supports the hypothesis. Apparently, it becomes harder to match the elements mentioned in the question with the elements in the graphical model when the text labels get larger.

Table 1. Sum of CORRECTAnswer for the two model variants (TEXT $=0$ is abstract letters and TEXT $=1$ textual labels

\begin{tabular}{|r|cc|r|}
\hline TEXT & correct all & $\%$ \\
\hline 0 & 608 & 828 & $73.43 \%$ \\
1 & 493 & 684 & $72.08 \%$ \\
\hline
\end{tabular}




\subsection{Prediction of Correct Answers}

Up to now, we have studied bivariate correlations between different hypothetical factors and different score variables as proxies for understandability. In this section we investigate the combined capability of the factors to explain the variance of the dependent variable CORRECTANSWER. This variable captures whether a single question related to a model was answered correctly by a participant. As the dependent variable is binary, we use a logistic regression (logit) model. The idea of a logit model is to model the probability of a binary event by its odds, i.e., the ratio of event probability divided by non-event probability. These odds are defined as $\operatorname{logit}\left(p_{i}\right)=\ln \left(\frac{p_{i}}{1-p_{i}}\right)=B_{0}+B_{1} x_{1, i}+\ldots+B_{k} x_{k, i}$ for $k$ input variables and $i$ observations, i.e. EPC $i$ in our context. From this follows that

$$
p_{i}=\frac{e^{B_{0}+B_{1} x_{1, i}+\ldots+B_{k} x_{k, i}}}{1+e^{B_{0}+B_{1} x_{1, i}+\ldots+B_{k} x_{k, i}}}
$$

The relationship between input and dependent variables is represented by an S-shaped curve of the logistic function that converges to 0 for $-\infty$ and to 1 for $\infty$. The cut value of 0.5 defines whether event or non-event is predicted. $\operatorname{Exp}\left(B_{k}\right)$ gives the multiplicative change of the odds if the input variable $B_{k}$ is increased by one unit, i.e. $\operatorname{Exp}\left(B_{k}\right)>1$ increases and $\operatorname{Exp}\left(B_{k}\right)<1$ decreases error probability.

The significance of the overall model is assessed by the help of two statistics. Firstly, the Hosmer \& $\&$ Lemeshow Test should be greater than 5\% to indicate a good fit based on the difference between observed and predicted frequencies [33]. Secondly, Nagelkerke's $\mathrm{R}^{2}$ ranging from 0 to 1 serves as a coefficient of determination indicating which fraction of the variability is explained [34]. Furthermore, each estimated coefficient of the logit model is tested using the Wald statistic, for being significantly different from zero. The significance should be less than $5 \%$. We calculate the logistic regression model based on a stepwise introduction of those variables that provide the greatest increase in likelihood. For more details on logistic regression, see [33].

Figure 5 shows the result of the logistic regression estimation. The best model is derived in step 4 since it covers the largest set of variables such that all have a significance in the Wald statistic better than $5 \%$. The Nagelkerke's $\mathrm{R}^{2}$ for this step 4 model is 0.293 indicating that quite a considerable share of the overall variance can be explained. Still, the Hosmer $\&$ Lemeshow Test is below $5 \%$ which signals that there seem to be other factors that are not covered by the model. The model includes four variables, namely THEORY, SEPARABILITY, TEXTLENGTH, and DURATiOn. While TEXTLENGTH appears to have a negative effect on CORRECTANSWER as expected by $\mathbf{C 2}$, the other variables have a positive impact on the correctness of answering a question. This confirms the hypotheses $\mathbf{P 1}$ and M2. It is interesting to note that the step 4 model includes factors that are related to all three influences that we identified, i.e. personal, model, and content factors with the model factor SEPARABILITY having the greatest relative impact. 
Variables in the Equation

\begin{tabular}{|c|c|c|c|c|c|c|c|}
\hline & & B & S.E. & Wald & df & Sig. & $\operatorname{Exp}(B)$ \\
\hline Step $1^{a}$ & THEORY & .148 & .009 & 300.694 & 1 & .000 & 1.160 \\
\hline \multirow[t]{2}{*}{ Step $2^{b}$} & SEPARABILITY & 1.012 & .384 & 6.966 & 1 & .008 & 2.752 \\
\hline & THEORY & .121 & .013 & 86.815 & 1 & .000 & 1.129 \\
\hline \multirow[t]{3}{*}{ Step $3^{c}$} & TEXTLENGTH & -.001 & .000 & 2.831 & 1 & .092 & .999 \\
\hline & SEPARABILITY & 1.088 & .387 & 7.908 & 1 & .005 & 2.969 \\
\hline & THEORY & .133 & .015 & 80.452 & 1 & .000 & 1.142 \\
\hline \multirow[t]{4}{*}{ Step $4^{d}$} & TEXTLENGTH & -.001 & .000 & 3.946 & 1 & .047 & .999 \\
\hline & SEPARABILITY & .935 & .396 & 5.582 & 1 & .018 & 2.548 \\
\hline & THEORY & .120 & .016 & 52.634 & 1 & .000 & 1.127 \\
\hline & DURATION & .006 & .003 & 3.073 & 1 & .080 & 1.006 \\
\hline \multirow[t]{5}{*}{ Step $5^{e}$} & TEXTLENGTH & -.001 & .000 & 2.815 & 1 & .093 & .999 \\
\hline & DIAMETER & -.015 & .010 & 2.493 & 1 & .114 & .985 \\
\hline & SEPARABILITY & 1.185 & .428 & 7.677 & 1 & .006 & 3.270 \\
\hline & THEORY & .140 & .021 & 45.103 & 1 & .000 & 1.150 \\
\hline & DURATION & .008 & .004 & 4.601 & 1 & .032 & 1.008 \\
\hline
\end{tabular}

a. Variable(s) entered on step 1: THEORY

b. Variable(s) entered on step 2: SEPARABILITY

c. Variable(s) entered on step 3: TEXTLENGTH

d. Variable(s) entered on step 4: DURATION

e. Variable(s) entered on step 5: DIAMETER

f. Stepwise procedure stopped because removing the least significant variable result in a previously fitted model.

Fig. 5. Logistic regression function estimated for the sample

\section{Conclusions}

In this paper we have used an online questionnaire to acquire insight into the empirical connection between different influential factors and process model understandability. In particular, we focused on several personal, model, and content related impact factors. By the help of a correlation analysis we found that the personal factor THEORY (0.491), the model factor SEPARABILITY (0.886 Spearman), and the content factor TEXTLENGTH (-.836) were significantly correlated with the different proxies for understandability. A multi-variate logistic regression analysis confirmed the importance and impact direction of these three variables, and also included DURATION in the statistical model. Altogether, the survey supports the hypothesis that personal, model, and content related factors influence the understandability of business process models. This has strong implications for business process modeling initiatives in organizations. First, there is apparently a need for guidelines that lead to understandable process models in terms of structure and text labels. Second, there is a need for training since experienced modelers perform better in understanding.

The research design used in this paper has some limitations. Firstly, we only investigated three categories of influence factors of understandability. Future research will have to analyze other categories as well. Given the considerable 
number of impact factors that might be important ([9] mention at least six) it will be difficult to study the relative importance of these factors. Secondly, our experiment covered only a limited set of six models and a limited set of 42 participants. Future surveys need to have more models tested by each participant for comprehending the relative importance of the different model metrics. This implies challenges with motivation and fatigue. Finally, more participants are needed to clearly identify which personal factors have the greatest impact on understanding a process model.

\section{Acknowledgement}

The author would like to thank Wolfgang Hammel, Gerald Schneider, Yvonne Tima, and Maria Weiler for their help in designing the questionnaire and conducting the experiment.

\section{References}

1. Hoppenbrouwers, S.S., Proper, H.E., van der Weide, T.: A Fundamental View on the Process of Conceptual Modeling. In: ER 2005, Proceedings. LNCS 3716. (2005) $128-143$

2. Becker, J., Rosemann, M., v. Uthmann, C.: Guidelines of Business Process Modeling. In van der Aalst, W., Desel, J., Oberweis, A., eds.: Business Process Management. LNCS 1806. (2000) 30-49

3. Moody, D.: Theoretical and practical issues in evaluating the quality of conceptual models: current state and future directions. Data \& Knowl. Eng. 55 (2005) 243-276

4. Davies, I., Green, P., Rosemann, M., Indulska, M., Gallo, S.: How do practitioners use conceptual modeling in practice? Data \& Knowl. Eng. 58 (2006) 358-380

5. Mendling, J., Moser, M., Neumann, G., Verbeek, H., Dongen, B., Aalst, W.: Faulty EPCs in the SAP Reference Model. In S. Dustdar, J.F., Sheth, A., eds.: BPM 2006, Proceedings. LNCS 4102. (2006) 451457

6. Mendling, J., Verbeek, H., Dongen, B., Aalst, W., Neumann, G.: Detection and Prediction of Errors in EPCs of the SAP Reference Model. Data \& Knowl. Eng. (2007) accepted for publication.

7. Simon, H.: Sciences of the Artificial. 3rd edn. The MIT Press (1996)

8. Mendling, J.: Detection and Prediction of Errors in EPC Business Process Models. $\mathrm{PhD}$ thesis, Vienna University of Economics and Business Administration (2007)

9. Mendling, J., Reijers, H., Cardoso, J.: What makes process models understandable? In Alonso, G., Dadam, P., Rosemann, M., eds.: BPM 2007, Proceedings. LNCS 4714. (2007) 48-63

10. Lindland, O., Sindre, G., Sølvberg, A.: Understanding quality in conceptual modeling. IEEE Software 11 (1994) 42-49

11. Krogstie, J., Sindre, G., Jørgensen, H.: Process models representing knowledge for action: a revised quality framework. Europ. J. of Inf. Systems 15 (2006) 91-102

12. Moody, D., Sindre, G., Brasethvik, T., Sølvberg, A.: Evaluating the quality of process models: Empirical testing of a quality framework. In Spaccapietra, S., March, S.T., Kambayashi, Y., eds.: ER 2002, Proceedings. LNCS 2503. (2002) 380-396 
13. ISO: Information technology - software product evaluation - quality characteristics and guide lines for their use. ISO/IEC IS 9126 (1991)

14. Güceglioglu, A.S., Demirörs, O.: Using software quality characteristics to measure business process quality. In van der Aalst, W., Benatallah, B., Casati, F., Curbera, F., eds.: BPM 2005, Proceedings. LNCS 3649. (2005) 374-379

15. Gemino, A., Wand, Y.: Evaluating modeling techniques based on models of learning. Commun. ACM 46 (2003) 79-84

16. Green, T., Petre, M.: Usability analysis of visual programming environments: A 'cognitive dimensions' framework. J. Vis. Lang. Comput. 7 (1996) 131-174

17. Lee, G., Yoon, J.M.: An empirical study on the complexity metrics of petri nets. Microelectronics and Reliability 32 (1992) 323-329

18. Nissen, M.: Redesigning reengineering through measurement-driven inference. MIS Quarterly 22 (1998) 509-534

19. Morasca, S.: Measuring attributes of concurrent software specifications in petri nets. In: METRICS '99, Proceedings (1999) 100-110

20. Reijers, H., Vanderfeesten, I.: Cohesion and coupling metrics for workflow process design. In Desel, J., Pernici, B., Weske, M., eds.: BPM 2004, Proceedings. LNCS 3080. (2004) 290-305

21. Cardoso, J.: Evaluating Workflows and Web Process Complexity. In: Workflow Handbook 2005. (2005) 284-290

22. Balasubramanian, S., Gupta, M.: Structural metrics for goal based business process design and evaluation. Business Process Management Journal 11 (2005) 680-694

23. Canfora, G., García, F., Piattini, M., Ruiz, F., Visaggio, C.: A family of experiments to validate metrics for software process models. Journal of Systems and Software 77 (2005) 113-129

24. Aguilar, E.R., Ruiz, F., García, F., Piattini, M.: Towards a Suite of Metrics for Business Process Models in BPMN. In Manolopoulos, Y., Filipe, J., Constantopoulos, P., Cordeiro, J., eds.: ICEIS 2006, Proceedings (III). (2006) 440-443

25. Laue, R., Gruhn, V.: Complexity metrics for business process models. In Abramowicz, W., Mayr, H.C., eds.: BIS 2006, Proceedings. LNI 85. (2006) 1-12

26. Cardoso, J.: Process control-flow complexity metric: An empirical validation. In: IEEE SCC 06, Proceedings. (2006) 167-173

27. Rosemann, M., Recker, J., Indulska, M., Green, P.: A study of the evolution of the representational capabilities of process modeling grammars. In Dubois, E., Pohl, K., eds.: CAiSE 2006, Proceedings. LNCS 4001 (2006) 447-461

28. Agarwal, R., Sinha, A.: Object-oriented modeling with UML: a study of developers' perceptions. Communications of the ACM 46 (2003) 248-256

29. Sarshar, K., Loos, P.: Comparing the control-flow of epc and petri net from the end-user perspective. In Aalst, W., Benatallah, B., Casati, F., Curbera, F., eds.: BPM 2005, Proceedings. LNCS 3649. (2005) 434-439

30. Lange, C., Chaudron, M.: Effects of defects in uml models: an experimental investigation. In Osterweil, L., Rombach, H., Soffa, M., eds.: ICSE 2006, Proceedings. (2006) 401-411

31. Mendling, J., Aalst, W.: Towards EPC Semantics based on State and Context. In M. Nüttgens and F.J. Rump and J. Mendling, ed.: EPK 2006, Proc. (2006) 25-48

32. Mendling, J., Aalst, W.: Formalization and Verification of EPCs with OR-Joins Based on State and Context. In Krogstie, J., Opdahl, A., Sindre, G., eds.: CAiSE 2007, Proceedings. LNCS 4495. (2007) 439-453

33. Hosmer, D., Lemeshow, S.: Applied Logistic Regression. 2nd edn. (2000)

34. Nagelkerke, N.: A note on a general definition of the coefficient of determination. Biometrika 78 (1991) 691-692 